\title{
The role of EL2 in the infrared transmission images of defects in semi-insulating GaAs
}

\author{
Seong-Jun Kang, Sung-Seok Lee, Member, KIICE
}

\begin{abstract}
Infrared transmission images from GaAs semi insulating wafers were considered for years as directly related to the quantum absorption by electrons on fundamental states of deep centers, especially EL2. The satisfying correspondence of these images with the dislocations revealed by etching or $X$ ray topography or infrared tomography led to the opinion that a strong concentration of EL2 centers was to be expected in the immediate vicinity of the dislocations. More recent work indicates that contrary to the expected behavior the photoquénching of transmission images at $T=80 \mathrm{~K}$ does not appreciably change the image structure itself but more largely the uniform background level of absorption. Such investigations show that the transmission images of isolated dislocations (Indium doped materials) or cell structures of tangled dislocations (undoped materials) can be partly attributed to scattered light; similar operation at $T=$ $10 \mathrm{~K}$ removes the dark features associated to EL2 but still preserves the skeleton of the pattern which is due to scattering. A result of the measurements is that dislocations must not be considered any longer as inexhaustive EL2 reservoirs. The lifetime of the photoquénching mechanism is shown to vary differently for EL2 centers located close to the dislocations or in the matrix. In this paper we will develop the details of infrared image photoquénching experiments in the vicinity of dislocations; undoped and In doped GaAs materials will be shown. These results will be discussed in the light of surface etching experiments.
\end{abstract}

Index Terms-EL2, GaAs, Infrared, Semi-Insulating

\section{INTRODUCTION}

IT is known that electrical compensation of residual $\left(\mathrm{N}_{\mathrm{A}}-\mathrm{N}_{\mathrm{D}} \approx 10^{15} \mathrm{~cm}^{-3}\right)$ unintentional doping of GaAS SI materials is provided by the deep donor EL2, the nature of which is still uncertain[1]. Local variations of the densities of these centers are expected to induce local fluctuations of potential which detrimentally affect the deviation of the MESFETs specifications[2].

This is supposed to occur specifically in the regions close to dislocation clusters even though large deviations

Manuscript received October 13, 2011; revised November 21, 2011; accepted November 30, 2011.

Seong-Jun Kang is with the Dept. of Information and Communication Engineering, Mokpo National University(sjkang@mokpo.ac.kr)

Soeng-Seok Lee is with the Dept. of Information and Communication Engineering, Mokpo National University(slee@mokpo.ac.kr) have also been found in FETs implanted in "dislocation free" (DF) materials. Undoped LEC grown materials usually display a typical pattern related to dislocations entangled to make "walls" and "cells": these features can also be obtained by chemical etching or by X ray topography or by infrared transmission (IRT). The latter technique is simple to achieve when using image processing but the physical origin of the image is still unsatisfactorily explained. The IRT images have been traditionally interpreted by the optical absorption related to the fundamental transition on $\mathrm{EL} 2^{0}$ centers even though a strong scattering contribution is also to be considered[3].

The IRT images have a low contrast; the blurred pattern gives rise to a poor image if compared for instance with Laser Scattering Tomography(LST) images[4]. So an important technological point is to appreciate the specific contribution of the EL2 absorption in the EL2 absorption in the image in order to calibrate the local EL2 density.

On the other hand, EL2 is a complex center and some workers have suggested that it could belong to a series of similar but nonetheless different species and also that its configuration could change depending on thermal, optical and electrical history[5],[6]. This center is also able to be converted into a stable and inactive configuration if illuminated with white light at low temperature. This photoquenching(PQ) phenomenon is considered as the very finger print of the sample[7] which can be thermally regenerated if heated above a threshold temperature of some $T_{\mathrm{th}}=125 \mathrm{~K}$. Brozel and Stirland[8] initially found that the IRT image disappear after $\mathrm{PQ}($ at $\mathrm{T}=12 \mathrm{~K}$ ) but later Castagné [3] also showed that above $\mathrm{T}=80 \mathrm{~K}$ only the background of the IRT image was affected by PQ whereas the cell pattern and relative contrast was kept unchanged[2],[5],[6].

This paper deals with a comparative analysis of IRT images at $T_{1}=10 \mathrm{~K}$ and $T_{2}=90 \mathrm{~K}$ before and after PQ in order to establish a relation between EL2 and the dislocations and also to discriminate the behaviours of the different EL2 centers. It will be shown that PQ mechanisms are not identical for all EL2: some(especially located in the matrix)have a short lifetime and are converted at temperatures up to some $120 \mathrm{~K}$, others (especially that located in the walls) are more stable and require a much lower temperature (below $80 \mathrm{~K}$ ). Nevertheless the regeneration mechanism at $\mathrm{T}>125 \mathrm{~K}$ holds the same for all EL2. 


\section{INFRARED IMAGE ANALYSIS}

From the initial measurements[3] of light scattering in GaAs and also the subsequent developments in LST[9] it would appear that this mechanism of contrast generation should be taken into account in the IRT image formation as well as absorption itself. It is likely that a Rayleigh type scattering prevails, the main scatterers being microprecipitates located along the path of grown in dislocations[4].

Depending upon the image magnification, the relative importance of the scattering with respect to the absorption varies to such an extent that macroscale images[10] could be considered as essentially due to absorption whereas microscale images are mainly related to the scattering[3] on condensed particles. The main reason for this is the smooth variation of the EL2 densities (blurred images) in comparison with the steep variation of scattering on sharp objects.

PQ obviously modifies the absorption contribution whereas the scattering would not be changed appreciably if considered as independent of EL2; this means that after PQ the pattern of decorated dislocations still has to remain in the image if the contrast specification of the set up is kept high enough.

The initial work was done by Brozel[8] showing a bleaching of the IRT image at $\mathrm{T}=12 \mathrm{~K}$ after PQ but these results were found to be different with further experiments by Castagné[3] who showed that PQ for $120>\mathrm{T}>80 \mathrm{~K}$ only reduces the background absorption but in no way the pattern of the image; as long as EL2 is considered as typified by a regeneration threshold at $T_{\text {th }}$ this result leads to the contradictory conclusion that EL2 is a microscopic defect quasi uniformly distributed in the bulk (fourfold symetry) and quite independent of the dislocations.

From these PQ experiment quantitative evaluation of $\mathrm{EL}^{0}$ densities $\delta$ were performed in both undopedand In doped[11] materials; the former (see Fig.1 a-b-c) indicated a lower in the walls with respect to the cell interior (in contradiction with Brozel[8]) and an intermediate zone of enhanced $\bar{b}$; nevertheless the spatial variations of $\bar{\delta}$ is weak $\pm 20 \%$ ) discounting the idea of considering dislocations as very large reservoirs for EL2 .

The latter showed (Fig. 2. a-b-c) a different behavior: isolated $<001>$ dislocations found in the midradius region of the wafers, appear to be surrounded by large (500 to $700 \mu \mathrm{m}$ wide) cylindrical

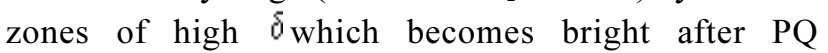
whereas the outside region is not affected.

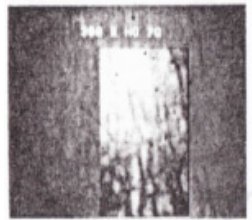

(a)

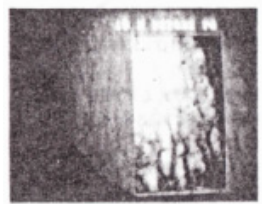

(d)

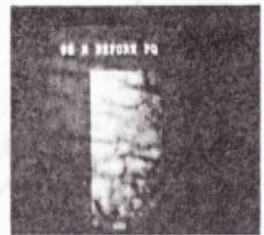

(b)

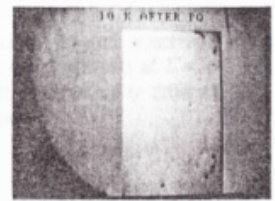

(e)

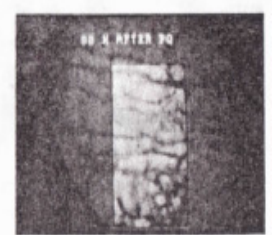

(c)
Fig. 1. IRT images of an undoped LEC grown GaAs material (Cmoinco) at room temperature (a), at $\mathrm{T}=90 \mathrm{~K}$ before (b) and after (c) $\mathrm{PQ}$, at $\mathrm{T}=10 \mathrm{~K}$ before (d) and after PQ (e). Boxes correspond to processed area of interest.

In the central part of the wafer (core) where dislocations are more tightly packed these bright zones largely overlap to give rise to a large uniform zone (Fig. 3) of high $\bar{d}$. A puzzling point is that these zones cannot be distinguished before $P Q$, thus indicating a similar density of active EL2 ${ }^{0}$. It would be very surprising that two different kinds of similar EL2 centers could be distributed in exactly complementary densities all over the wafer. From this it was concluded that more detailed PQ image analysis at lower temperature is required to achieve a better understanding.

\section{LOW TEMPERATURE PHOTOQUENCHING}

The experimental set up uses an Oxford Helium cryostat flushed with refined $\mathrm{He}$ in order to remove perturbations of residual gas condensation on the sample in the critical $70-90 \mathrm{~K}$ temperature range.

The samples were a Cominco undoped SI LEC sample and an In doped SI LEC sample (grown by LETI). Both are considered as typical, they are $400 \mu \mathrm{m}$ thick and double face polished.

Complete results of PQ at $90 \mathrm{~K}$ and $10 \mathrm{~K}$ are reported in Fig. 1 and Fig. 2. The IRT probe light is filter limited above $0.9 \mu \mathrm{m}$ and it is kept low enough not to induce any unwanted PQ; the sample is kept in the dark between each image. The PQ is carried out by a white light illumination during a matter of minutes and it is verified that saturation is reached. Adapted image processing facilities also allows to improve contrast resolution as shown in the boxes in the figures 1 and 2 . 


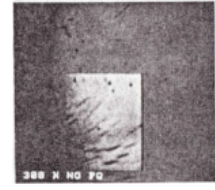

(a)

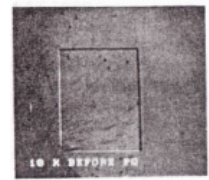

(d)

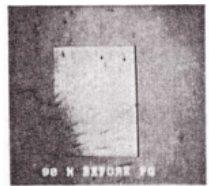

(b)

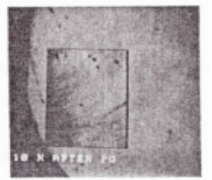

(e)
Fig. 2. IRT images of an In doped LEC grown GaAs material (LETI) at room temperature(a). at $\mathrm{T}=90$ $\mathrm{K}$ before (b) and after (c) $\mathrm{PQ}$, at $\mathrm{T}=10 \mathrm{~K}$ before (d) and after PQ (e). Boxes correspond to computer processed area of interest.

At $\mathrm{T}=10 \mathrm{~K}$ unlike at $\mathrm{T}=90 \mathrm{~K}$ the PO strongly affects the cell pattern of the IRT image in undoped samples but contrast stretching still reveals the cell "skeleton" (Fig. 1) due to light scattering on dislocation precipitates : this can be considered as the ultimate contribution of scattering in the IRT image which is still observable after PQ as required. Nevertheless the contrast is severely reduced which means that a wealth of EL2 centers (especially those located close to the dislocations) require a temperature below $90 \mathrm{~K}$ to be quenched, with a reasonable time constant: the most satisfying speculation from this observation is that PQ mechanism involves the participation of a carrier trapped on an auxiliary center which is not the same for EL2 in the matrix (deep level $\mathrm{E}_{1}>150 \mathrm{meV}$ ) or for EL2 close to the dislocations (shallow level $\mathrm{E}_{2}<80 \mathrm{meV}$ ).

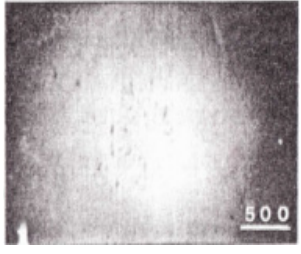

(a)

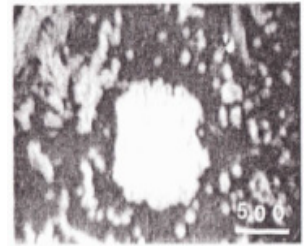

(b)
Fig. 3. Macroscale view of the In doped sample at $\mathrm{T}=90 \mathrm{~K}$ before (a) and after (b) PQ.

In Indium doped samples individual decorated dislocations can be observed (Fig. 2) but PQ at $10 \mathrm{~K}$ no longer induces the bright zones observed at $90 \mathrm{~K}$; it could be concluded that the bright zones (which extend to the whole wafer when dislocations are concentrated) correspond to $E_{1}$ impurity whereas DF zones corresponds to a shallower level $\mathrm{E}_{3}$. Such a model is consistent with a unique relaxation mechanism internal to EL2 and thermally assisted above $\mathrm{T}_{\text {th }}$ where $\mathrm{As}^{+}{ }_{\mathrm{Ga}}$ is regenerated[1].

This hypothesis of variable microscopical chemical doping was confirmed by electrical measurements on small contacts $(150 \mu \mathrm{m})$ selectively located in the walls (W) or in the center of large cells (C); photoconductivity transients at $\mathrm{T}=4 \mathrm{~K}$ and also thermally stimulated conductivity (TSC) spectra were shown to be drastically different in (W) or in (C)[12].

\section{CONCLUSIONS}

From these experiments it follows that the discrepancies between the PQ results of Skolnick and Castagné are now satisfactorily bridged: the scattering residual part of the image is obtained but a non negligible absorption of EL2 is also found close to dislocations (less than 50\% variation); these centers need much lower PQ temperature and longer time constants in order to be converted but their regeneration process is not modified.

The exact role of the external defect levels $E_{1}, E_{2}, E_{3}$ is playing in the PQ mechanism is to be explained but these levels cannot be confused with the EL2 internal shallow level neither with the shallow impurities responsible of the "reverse contrast" [11],[13]. Participation of carbon atoms can likely be ruled out because it was shown that it should be uniformly distributed in cells and walls.

It can be suggested that the chemical local composition should strongly vary in the dislocation environment and in the matrix as suggested by the various micro precipitates discovered by LST techniques[4].

\section{REFERENCES}

[1] H.J. von Bardeleben, "Metastable state of EL2 defect in GaAs, phys. Rev. B, vol. 40, no. 18, pp. 12546-12549, 1989.

[2] J.P. Fillard, "Reconnaissance des défauts et traitementd images pour les composés III- $V$ ", Annales des Télécommunications vol. 42, no. 3-4, mars-avril, pp. 149-180, 1987.

[3] Castagné"EL2 related levels in GaAs-SI transmission and dispersion in infrared imaging", solid state commun. 54, 653, 1985

[4] P. Gall, J.P Fillard, M Castagné, "Microtomography observation of precipitates in semi-insulating GaAs materials", Jour. Appl. Phys. 64, pp. 5161-5167, 1988

[5] J.P. Fillard, J. Bonnafé, M. Castagné, Jour. Appl. Phys. 56, pp. 3020-3025, 1984.

[6] J.P. Fillard, J. Bonnafé, M. Castagné, Jour. Appl. Phys. Let. A35, pp149-152, 1984.

[7] L. Samuelson and P. Omling, "Optical properties of EL2 in GaAs", Phys. Rev. B 34, pp. 5603-5608, 1986

[8] M.R. Brozel, I. Grant, D.J. Stirland, "Direct observation of the principal deep level (EL2) in undoped semi-insulating GaAs", Appl. Phys. Lett. 42(7), April, pp. 610-612, 1983.

[9] Ogawa T DRIP I symposium Mat Sc. Monographs 311 J.P. Fillard Ed, 1985.

[10] M.S. Skolnick and M.R. Brozel, "Distinction between near infrared optical absorption and light scattering in semi-insulating GaAs", Appl. Phys. Lett. 48(5), February, pp. 341-343, 1986.

[11] S.J.Kang, "Contribution àl'étude du center EL2 dansGaAs semiisolant par photoextinction des images de transmission infrarouge”, Ph.D dissertation, U.S.T.L.(Montpellier II ), France, 1990.

[12] J.C. Parker and R. Bray, "Analysis of photoassisted thermal 
recovery of metastable EL2 defects in GaAs", Phys. Rev. B, 15 April, pp. 6368 6376, 1988.

[13] Hans J. Queisser, "Reversal of contrast for infrared absorption of deep levels in semi-insulating GaAs", Appl. Phy. Lett. 46(8), 15 April, pp 757 759, 1985

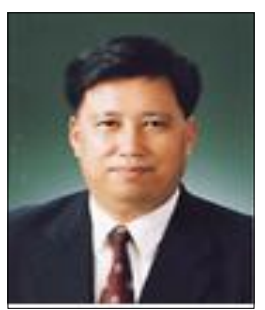

Seong-Jun Kang was born in Wando Korea on April 4. He received the B.S. degree in electronics engineering from Ajou University, Suwon Korea in 1978 and the M.S. degree in electronics engineering from Yonsei University, Seoul Korea in 1981. He holds the D.E.A. and Doctorat (Ph.D) degrees in electronics engineering from l'Université des Sciences et Techniques duLangeudoc (Langeudoc University of science and Technology), Montpellier France. From 1980 to 1994, he was a chief researcher in the departments of telecommunications standardization and semiconductor technology at Electronics and Telecommunications Research Institute (ETRI). He joined the department of information engineering at the Mokpo National University in 1994 as a full time instructor, where he is currently a professor. His research interests are in the areas of infrared imaging technology, infrared communication for indoor applications and electro-optical systems.

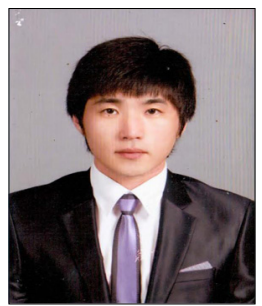

Seong-Seok Lee was born in mokpo Korea on Jun 17. He received the B.S. degree in information and communication engineering from Mokpo University, Muan Korea in 2008. His research interests are in the areas of infrared imaging technology, infrared communication for indoor applications and electro-optical systems 